\title{
¿Qué pasa si hábitats y especies van desapareciendo?
}

\author{
Miguel Aymerich Huygues-Despointes ${ }^{1}$
}

Sumario: I. LAS GRANDES CIFRAS: EL COSTE DE LA PÉRDIDA DE BIODIVERSIDAD A ESCALA PLANETARIA. II. DESAPARICIÓN DE ESPECIES COMERCIALES. 1. El colapso de la pesquería de bacalao en las costas atlánticas canadienses. 2. La anchoa cantábrica. III. EJEMPLOS DE RECUPERACIÓN IN EXTREMIS DE ESPECIES. 1. Una historia de éxitos: el caimán de Luisiana. 2. El efecto sinérgico de la recuperación de especies en peligro: los carneros de Pakistán. IV. CASO HIPOTÉTICO: ¿Y SI SE HUBIERA EXTINGUIDO UNA ESPECIE BANAL? 1. El tunicado Ecteinascidia turbinata.

En el año 48 adC, durante la guerra entre Roma y Egipto, tuvo lugar una terrible batalla entre la flota egipcia y la romana, frente a la Ciudad de Alejandría. Como consecuencia de la contienda, se produjo un enorme incendio en la ciudad que afectó a casi toda el área urbana y también al edificio del Museo donde estaba ubicada la gran biblioteca, depositaria de todas las copias del antiguo Occidente.

Toda la riqueza intelectual, todo el saber acumulado durante siglos desapareció en unas horas, convertido en humo y cenizas. Sólo algunos rollos de papiro, de un número original cercano al millón, pudieron salvarse, ante la indiferencia de la población de la ciudad, incapaz de comprender la importancia el tesoro que se cobijaba en ese edificio.

Más de dos milenios después, el incendio de la Biblioteca de Alejandría sigue recordándose como una pérdida irreparable para la humanidad, Sin embargo, si alguien se preguntara qué coste pudo tener la pérdida del saber acumulado durante siglos, o incluso milenios, la respuesta sería francamente difícil o tal vez incluso imposible. Si,

1 Subdirector General Adjunto de Evaluación de Impacto Ambiental. Ministerio de Medio Ambiente y Medio Rural y Marino. 
a falta de un inventario de sus existencias, no sabemos ni remotamente lo que se almacenaba en sus estanterías, las diversas materias que se trataban, las aplicaciones prácticas que podían contener los rollos quemados, cómo calcular lo que pudo suponer la desaparición casi completa de la gran y mítica biblioteca?

Algo similar ocurre cuando abordamos la pérdida de biodiversidad que está sufriendo la Tierra. Nuestro planeta ha ido acumulando durante cientos de millones de años, y mediante complicados procesos evolutivos, un inmenso bagaje de biodiversidad, consistente en formas de vida de todo tipo, que habitan desde las simas oceánicas profundas hasta las montañas más elevadas, pasando por las profundidades de los hielos de la Antártida, ocupando y colonizando la totalidad de ambientes y situaciones, incluso las más extremas que se producen en el globo.

Tantas formas de vida que, a pesar de los muchos esfuerzos desarrollados durante siglos por los científicos y de la tecnología cada vez más desarrollada de la que disponemos, aún conocemos sólo una mínima fracción de las que existen en nuestro mundo. Si somos aún incapaces de saber cuantos vertebrados, los seres más grandes y visibles, viven en nuestro planeta, si cada expedición científica a lugares remotos tiene como resultado la descripción de decenas o cientos de nuevas especies, qué podemos decir si nos fijamos en los pequeños organismos invisibles al ojo humano? Aquí, el desconocimiento es tan grande que se podría afirmar que el trabajo está aún por hacer en su casi totalidad.

Edward Wilson, el científico estadounidense que para muchos es el padre del concepto biodiversidad, que define como el conjunto de especies vivas del planeta, su variabilidad genética y sus ecosistemas, señaló hace años "La Tierra es un planeta muy mal conocido, el hombre tan solo ha descrito 1,8 millones de especies de las entre 10 y 30 millones que calculamos que existen; un solo gramo de suelo fértil contiene 5.000 tipos de bacterias". Y añadió: "Cualquiera de ustedes Ileva enganchadas a las suelas de sus zapatos cantidades de especies desconocidas".

Falta por tanto aún mucho tiempo para que el "bibliotecario de la biodiversidad" tenga un inventario completo de lo que nuestro planeta, nuestra gran Biblioteca de Alejandría de la vida, alberga en tierras, mares y atmósfera.

Sabemos por supuesto que el proceso evolutivo es dinámico y lleva a la extinción de muchas especies. Sólo entre el 2 y el $4 \%$ de las que vivieron en algún momento en la Tierra existen aún, pues la inmensa mayoría desapareció mucho antes de que la especie humana empezara a poblar el Planeta.

Pero lo que ocurre ahora es que las especies se están extinguiendo a un ritmo mucho más elevado que nunca, debido a la acción del hombre. Se han perdido al menos unas mil especies en los últimos 5 siglos, cifra que seguramente es mucho mayor ya que se refiere naturalmente sólo a organismos "visibles" y no a los invertebrados, 208 microorganismos.... El ritmo de las extinciones conocidas que ocurrieron en el último 
siglo es, aproximadamente, entre 50 y 500 (algunos autores menciona cifras de hasta 10.000) veces superior al ritmo de extinción calculado a partir del registro fósil, de 0,1 a 1 extinción por millón de especies por año. Y lo que es aún más grave, todas las predicciones auguran que estas tasas van a crecer muy significativamente en las próximas décadas. Nuestras "estanterías" han ido perdiendo existencias, en forma de especies extinguidas o de ecosistemas destruidos, a un ritmo cada vez más acelerado y este proceso se va a agravar en este siglo si no se toman medidas contundentes para revertirlo.

Pero si quisiéramos saber que supone esa pérdida desde el punto de vista económico -por no hablar de otros aspectos éticos, culturales, sociales, estéticos, recreativos, sentimentales...--, la respuesta no es casi nunca posible. ¿Cómo calcular la importancia de ese goteo cada vez más acelerado de pérdidas de especies si muchos de los seres que desaparecen ni han sido descritos, si no entendemos suficientemente su función en los ecosistemas, si es imposible imaginarnos el valor potencial que representan para el hombre? Esta dificultad explica, tal vez, la indiferencia con la que una gran parte de la humanidad escucha la voz de alarma de los científicos y conservacionistas, incapaz de entender, al igual que le ocurrió al pueblo de Alejandría, el desastre que supone para nuestro planeta, y para nosotros mismos, este empobrecimiento.

Aunque existe un número relativamente elevado de ejemplos de estudios sobre el valor de la biodiversidad y de los servicios que brindan los ecosistemas, así como de las consecuencias ecológicas de la desaparición de ecosistemas y especies, hay muy pocos trabajos rigurosos que se hayan ocupado de las consecuencias de la pérdida y/o degradación de la biodiversidad desde un enfoque económico. La aproximación simplista al problema sería decir que, una vez conocidos los servicios ambientales, la pérdida del ecosistema determina la desaparición de esos valores y nos priva de los beneficios que generan.

Pero las cosas no son tan sencillas cuando no estamos abordando la destrucción total del sistema, como podría ser la tala de un bosque primario, sino su sustitución por otro o su degradación parcial, situación en la que los servicios ambientales también se mantendrán en parte. También resulta complicado evaluar el efecto de la pérdida de una especie, a veces una cuestión casi irrelevante para el ecosistema en el que habita, que podrá seguir funcionando sin alteración alguna, al menos aparentemente; en otras ocasiones, por su posición estratégica en el engranaje, esta pérdida desencadena un efecto en cadena que conlleva la desaparición o degradación completa del sistema. Por ejemplo, la práctica desaparición de la nutria marina de muchas regiones donde habitaba, provocada por la sobrecaza, no sólo privó al hombre de este valioso recurso peletero. Al extinguirse la nutria, los erizos, parte muy significativa de su alimentación, se vieron libres de su principal predador, proliferaron sin control y eliminaron el kelp, un alga fundamental para el buen funcionamiento de todo el ecosistema, en especial para el alevinaje de los peces. La pesquería se resintió inmediatamente y la economía pesquera de la zona se vio pro- 
fundamente afectada, aunque por desgracia no se dispone de datos precisos sobre el valor de ese cambio.

Además, es necesario considerar el posible efecto de las "extinciones en cadena”. Un estudio publicado en la revista Science sostiene que cuando una especie está en peligro de extinción, hay cientos de especies más que corren el riesgo de desaparecer junto con ésta. Esto se debe a las relaciones de dependencia que existen entre ellas. Por cada animal o vegetal en peligro, los investigadores calculan que se debe temer la extinción adicional de unas 6.300 especies “dependientes". Según el estudio, esta reacción en cadena no había sido estudiada en detalle hasta el momento. Para sostener su argumento los científicos dan algunos ejemplos: la desaparición de la hormiga sudamericana Eciton burchelli o marabunta, podría significar a su vez el riesgo de extinción, al faltarles su alimento, de hasta cien especies de aves, escarabajos y ácaros.

Los análisis más fiables del coste económico de las extinciones, desgraciadamente en un número aún muy bajo, se han basado en casos de estudio en los que los ecosistemas o especies estaban siendo utilizados y explotados por el hombre, por lo que es posible conocer las consecuencias de su pérdida. Porque cuando tratamos de extinciones antiguas y de especies que no eran objeto de una utilización directa, la estimación de la pérdida es prácticamente imposible. ¿Cómo calcular lo que significó la extinción del dodó, el ave no voladora de la Isla Mauricio, extinguida por el hombre moderno? ¿Cuál hubiera sido su potencial económico en la sociedad actual? ¿Cómo calcular el valor potencial, aunque sea simplemente desde el punto de vista turístico, que hubiera constituido en nuestros días una colonia de focas monje en nuestras atestadas costas mediterráneas? ¿Qué rentabilidad, como recurso cinegético, se le podría sacar hoy a la paloma migratoria americana, cuya población superior a los 1.000 millones de ejemplares, fue aniquilada en unas pocas décadas por los colonizadores europeos? Son preguntas, y como estas podríamos formular ciento de ellas, sin respuesta alguna.

Tal vez por esta razón los casos más documentados se refieran a pérdidas de especies de interés pesquero, supuestos en los que es posible cuantificar con precisión la merma económica que produce su desaparición comercial. También algunas especies, que han estado a punto de extinguirse y que han podido ser recuperadas in extremis, aportan interesantes informaciones de los rendimientos que se hubieran podido perder. Finalmente, podemos conjeturar sobre lo hubiera pasado si se hubiera extinguido alguna especie concreta, incluso con organismos aparentemente sin ningún valor especial pero que albergan grandes potencialidades de desarrollo comercial.

Ante este general estado de desconocimiento, este artículo no pretende sino presentar una aproximación global pero somera a la cuestión y, sobre todo, aportar algunos ejemplos de pérdidas que sí han podido ser cuantificadas, porque se conocía 210 previamente la biodiversidad existente, sus funciones y su valor. 


\section{LAS GRANDES CIFRAS: EL COSTE DE LA PÉRDIDA DE BIODIVERSIDAD A ESCALA PLANETARIA}

En noviembre de 2007, se inició un estudio global, denominado COPI (Cost of Policy Inaction) dedicado a evaluar el coste de la inacción política frente a la pérdida de biodiversidad. El objetivo de este estudio era conseguir evaluar los beneficios y obtener una cifra global de la modificación del valor económico de la biodiversidad, desde la actualidad hasta 2050.

Gracias a la utilización de un modelo, se calculó como iba a evolucionar la biodiversidad en ese periodo: los principales indicadores describen, sobre todo, afecciones a la cantidad y calidad del suelo, y a la abundancia media de las especies de los ecosistemas, para todos los biomas del planeta. El modelo hace proyecciones regionales en lo referido a la conversión de bosques naturales en bosques explotados, así como de tierras agrícolas extensivas en intensivas, con la pérdida de naturalidad que resulta de estos dos fenómenos, que han sido tradicionalmente los más relevantes a lo largo de la historia. Sin embargo, la construcción de infraestructuras, la fragmentación de los habitats y el cambio climático son amenazas crecientes.

La pérdida de biodiversidad prevista para el año 2050 es del 10-15\%, y los ecosistemas más afectados serán muy probablemente las sabanas y los pastizales.

El escenario utilizado, que ha sido desarrollado en gran parte por la OCDE y se ajusta a otros de la FAO y de otras agencias de Naciones Unidas, prevé que la evolución del impacto sobre el suelo y de la biodiversidad se traducirá en cambios de los servicios de los ecosistemas, El principal problema que ha tenido el ejercicio ha sido atribuir un valor monetario al cambio de los servicios que brindan los ecosistemas, debido a la falta o a la disparidad de información disponible.

A pesar de las dificultades metodológicas, el estudio concluye que durante los primeros años de este periodo se perderán anualmente 50.000 millones de euros, y tan sólo considerando los ecosistemas terrestres. Hay que resaltar que estas cifras se refieren a Las "pérdidas de bienestar", ya que una gran parte de los beneficios no están incluidos en el PIB de los países.

La merma del capital natural no afecta únicamente al año en que se produce, sino que continúa en años sucesivos. Se calcula que estas pérdidas podrían alcanzar el 7\% del consumo anual en 2050.

Estas proyecciones han de ser consideradas con cautela, ya que se trata de modelos preliminares que deberán ser mejorados y ampliados. No obstante, ya se puede decir sin miedo a equivocarse que la pérdida de biodiversidad, al margen de cualquier otra consideración, supone un grave problema económico para la humanidad, seguramente aún más relevante para los más necesitados. 
Una vez presentados las grandes cifras la pérdida de biodiversidad en todo el Mundo, nos detendremos en algunos casos concretos y suficientemente documentados, que nos ayudarán a mejor entender el problema y la extrema complejidad de los cálculos necesarios para obtener información fiable.

\section{DESAPARICIÓN DE ESPECIES COMERCIALES}

\section{El colapso de la pesquería de bacalao en las costas atlánticas canadienses}

Aunque no trataremos aquí la desaparición total de una especie sino de su extinción comercial a escala regional, el siguiente ejemplo ilustra perfectamente las nefastas consecuencias de la mala gestión de un recurso, en la que la búsqueda de un provecho a corto plazo ha llevado a un desastre ecológico y humano, de carácter irreversible.

Cuando John Cabot alcanzó las costas de Newfoundland en 1497, las aguas de esta región estaban tan repletas de bacalao que el capitán escribió que "casi impedían la navegación de su barco". Durante los siguientes siglos la pesca de esta especie fue prácticamente la única razón por la que los hombres se acercaron y permanecieron en estas tierras desoladas.

Los canadienses, y las flotas llegadas de España y Portugal, pescaban por aquél entonces en aguas cercanas a la costa, usando una gran variedad de técnicas (trasmaIlos, nasas, palangres, pequeños arrastreros....). En el periodo que transcurre desde 1850 a 1950 se tienen documentadas capturas continuadas de unas 250.000 toneladas anuales en esta zona.

Sin embargo, a partir de 1950 se produjo un cambio de sistema de explotación, desde el artesanal, que había demostrado su sostenibilidad durante siglos, a uno técnicamente más avanzado y más "rentable”. Basándose en la tecnología de los barcos factoría utilizados para la caza de ballenas, los inmensos arrastreros/congeladores de países a veces muy distantes (Alemania, Reino Unido, Polonia, Cuba, la Unión Soviética, España, Portugal e incluso países asiáticos) se instalaron en la zona. Capaces de operar en aguas mucho más profundas y en las peores condiciones meteorológicas, estas factorías flotantes sometieron al stock a una presión jamás vista, alcanzando capturas de 800.000 t/año a finales de los años 50 y principios de los 60. A partir de ese momento se produjo un rápido declive del recurso, de manera que en 1975 los desembarcos eran ya inferiores a 300.000 toneladas.

Fuertemente alarmados por la rápida disminución de sus recursos pesqueros, Canadá y Estados Unidos promulgaron en 1976 nuevas leyes para extender su jurisdicción a las 200 millas náuticas, expulsando así a las flotas extranjeras a la "alta mar".

Lógicamente, con la desaparición de las flotas extranjeras las capturas descendie-

212 ron a su nivel más bajo, 139.000 toneladas en 1979. Pero en lugar de iniciar una política 
más conservadora y respetuosa, aplicando lo que hoy llamamos "el principio de precaución" la flota canadiense siguió cometiendo los mismos errores del pasado, e incrementó su capacidad extractiva hasta las 250.000 toneladas/ año a mediados de los 80 . Se siguió subvencionando con fondos públicos la construcción de los enormes arrastreros, cuyas inmensas redes lastradas por puertas y cadenas de metal literalmente "aran" el fondo marino, destruyendo todo a su paso y deteriorando irreversiblemente el ecosistema. Los arrastreros buscaban preferentemente las agrupaciones de reproductores, donde las capturas eran más jugosas, pero donde también se provocaba daños aún más significativos al stock y al ecosistema en su conjunto.

Durante la década de los 80 y a pesar de las primeras voces de alarma de los pescadores tradicionales, los cupos de captura se mantuvieron en las 250.000 toneladas/año. A partir del año 86 los científicos presentaron datos que demostraban el fuerte declive del stock y solicitaron una reducción de la cuota a la mitad. Después de años de análisis y contra-análisis (en los que no se produjo reducción de capturas) y de violentas polémicas entre los colectivos en contra y a favor de la regulación, en 1992 quedó finalmente demostrado que el stock estaba en unas condiciones absolutamente lamentables $y$, por primera vez en cuatrocientos años, se tuvo que prohibir la pesca del bacalao.

Con el cierre de la pesquería en 1992, 30.000 personas perdieron su empleo, tanto los pescadores como los de la industria transformadora. Posteriormente, el recorte de las cuotas en otras pesquerías (sobre las que se volcó parte del esfuerzo dedicado anteriormente al bacalao) incrementó esta cifra en 12.000 desempleados más. Las fábricas de conserva tuvieron que cerrar, los pesqueros se desguazaron o vendieron. Para amortiguar en lo posible el golpe provocado por el cierre de la pesquería, el Gobierno Federal tuvo que aportar 1.000 millones de dólares para pagar temporalmente el paro del sector pesquero, cantidad que habría de ser doblada en los años siguientes para sostener la economía tan maltrecha de la región.

Si la pesquería se hubiera mantenido en los valores que consiguió asegurar durante décadas, cuando se realizaba una gestión razonable del stock, a un precio tentativo de $3 € / \mathrm{Kg}$, se hubieran generado anualmente 750 millones de $€$ de ingresos directos, a los que habría que añadir todo el beneficio generado por la industria transformadora.

Sin embargo, casi veinte años después del cierre total de la pesquería, el stock de bacalao sigue siendo mínimo, probablemente porque la destrucción del ecosistema fue tan intensa que la especie ya no encuentra condiciones de vida mínimas para su supervivencia. De hecho, los peces pescados durante las campañas científicas muestran unas condiciones fisiológicas subóptimas.

Es muy improbable que esta especie pueda ser explotada comercialmente en el futuro, por lo que todo el sector económico que giraba a su alrededor y que generaría 
una cifra de negocio probablemente cercana a los 1000 millones de euros al año debe darse por definitivamente extinguido.

\section{La anchoa cantábrica}

La anchoa es una especie pelágica de pequeño tamaño y corta vida, que es objeto de explotación pesquera desde hace siglos por parte de la flota cantábrica. Vive y se desplaza en apretados cardúmenes, característica que favorece un nivel de capturas rentable incluso con bajos niveles poblacionales, debido a la existencia de modernos y efectivos métodos de detección de los bancos.

La pesca se realiza en la modalidad de cerco por parte de la flota española y mayoritariamente por arrastre pelágico por la francesa. El cerco se utiliza en primavera, cuando la anchoa, coincidiendo con la fase de puesta y reproducción, sube a la superficie, mientras que el arrastre se utiliza más en la segunda mitad del año y persigue a los peces a mayor profundidad.

Las capturas de anchoa del Golfo de Vizcaya han mostrado siempre amplias oscilaciones, una gráfica en diente de sierra. Desde mediados del siglo pasado, los máximos históricos se produjeron a mediados de los años 60, casi 90.000 toneladas/año, decreciendo a menos de 30.000 en el año 1975. A raíz de la introducción de nuevos mejoras tecnológicas (radar, sonar), las capturas volvieron a crecer durante unos años hasta las 50.000 toneladas, disminuyendo hasta niveles catastróficos en 1982, de sólo 5.000 toneladas.

A pesar de una pasajera recuperación en la década de los 90, la pesquería fue declinando progresivamente -17.000 t en 2000; 3.000 t en 2003, hasta que en el año 2005 se produjo el colapso. A pesar de haber permitido inicialmente unas capturas de 30.000 toneladas, la Comisión Europea tuvo que decretar el cierre de la pesquería, en vista del grave estado del stock. Este cierre se produjo tras varios años de discrepancias sobre la conveniencia o no del cierre entre los dos países implicados y tras múltiples avisos de los científicos, y a veces de los propios pescadores, sobre la gravedad de la situación.

A pesar del cierre prácticamente total de la pesquería desde esa fecha (en 2006 se capturaron poco más de 1.000 toneladas en una campaña experimental), el Centro Internacional para la Exploración del MAR (CIEM), órgano consultivo de la Unión Europea en materia de recursos marinos, ha recomendado el mantenimiento de la veda, al menos hasta julio de 2010 ya que la biomasa de anchoa no ha alcanzado aún el umbral mínimo necesario para la renovación de la especie. Lo que pase a partir de esa fecha es aún una incógnita pero es posible que la fecha de apertura de la pesquería aún deba posponerse.

Al intentar calcular las repercusiones de este cierre, empezaremos por decir que

214 las tripulaciones dedicadas en España a esta pesca combinaban unas 3.000 personas 
repartidas en los 90 barcos de la flota vasca, los 70 de la gallega, 60 de Cantabria y 12 de Asturias. El cierre de la pesquería sometió a estas tripulaciones a un cierre temporal de la actividad, que tuvo que ser apoyado y compensado, para minimizar los efectos sociales, con fondos públicos, con un montante de unos diez millones de euros/ año, es decir unos 50 millones en total (a los que se añadirán las cantidades que hayan de ser desembolsadas en el futuro para cubrir hipotéticas prórrogas de la veda).

En cuanto al valor del producto que no pudo ser pescado, si calculáramos el valor de la cuota permitida en los años anteriores a la veda, 30.000 t (si bien es cierto que esa cantidad no solía alcanzarse), a un precio medio de unos $3 € / \mathrm{kg}$, la pérdida

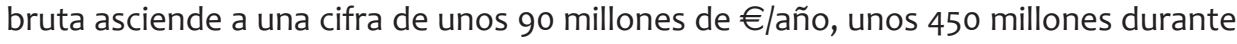
todo el periodo de veda.

Otras repercusiones indirectas, sobre todo las relacionadas con los empleos en tierra, principalmente en la industria conservera que emplea a varias miles de personas en estas regiones, son mucho más difíciles de medir ya que la escasez de pescado local ha sido en gran parte solventada con importaciones de otros mares.

Finalmente, existen otros impactos, desde el punto de vista económico y también biológico, imposibles de valorar por el momento, al menos de manera fiable, especialmente la repercusión de la escasez de anchoas en las poblaciones de predadores que se alimentan de esta especie (bonito, caballa, aves acuáticas de varias especies, cetáceos... ). La relación entre decaimiento del stock de anchoa y la situación de la población de bonito, otra especie sumamente importante para la flota cantábrica, no se conoce aún con suficiente detalle, por lo que es imposible saber si a los costes directos presentados anteriormente se deberían sumar los que genera un descenso de las capturas del túnido.

\section{EJEMPLOS DE RECUPERACIÓN IN EXTREMIS DE ESPECIES}

\section{Una historia de éxitos: el caimán de Luisiana}

Una de las historias que se suelen citar como buen ejemplo de la posibilidad de recuperar una especie y de obtener importantes beneficios de su explotación racional es la del caimán de Luisiana (Alligator mississippiensis).

Esta especie fue históricamente objeto de un alto nivel captura debido al elevado valor de su piel, lo que llevó a la población a un estado crítico en la década de los 60, hasta el punto de que en 1962 las autoridades del Estado tuvieron que vedar totalmente su caza.

Durante los diez años que siguieron se pusieron en marcha un gran número de trabajos científicos para mejorar el deficiente conocimiento de la especie, se diseña- 
ron metodologías fiables para el seguimiento de las poblaciones y se promulgaron disposiciones para permitir un futuro aprovechamiento sostenible.

En 1972 se concedieron permisos a 59 cazadores, con una cuota de captura de 1350 caimanes. Estas cifras se fueron incrementando paulatinamente en los años siguientes, regulándolas anualmente en función del estado de la población y de su tasa de reproducción. En 1975 ya se autorizaban 31.000 capturas a más de 1900 cazadores, y hasta 2007 se habían capturado más de 700.000 ejemplares.

A medida que las capturas se iban incrementando y el recurso se volvía más predecible, se fueron desarrollando otras actividades secundarias, además de la industria peletera. La carne se convirtió en un recurso muy valioso, el sector transporte se incrementó y se especializó para mejorar las condiciones de conservación de los productos derivados, el turismo incorporó partes de los caimanes en las tiendas de souvenir, hasta el punto de que todas las tiendas de Nueva Orleáns, una de las ciudades más turísticas de Estados Unidos, rebosan de productos realizados con partes de este animal (cabezas disecadas, mandíbulas....).

Por otra parte, paralelamente a la caza, a partir de los años 80 la cría en granjas, a partir de huevos recogidos en la naturaleza, experimentó un auge muy considerable. Como garantía de que la recolección de huevos no tendría un efecto desfavorable sobre las poblaciones silvestres, la administración ambiental obligó a los rancheros a liberar un número de juveniles igual al 17\% de los huevos recolectados -entre 300.000 y medio millón anual. Este porcentaje se ha ido reduciendo en años posteriores al comprobarse la buena salud de la población salvaje, hasta estabilizarse en el 12\% actual, pero supone no obstante la suelta anual de unos 60.000 ejemplares criados en cautividad, con unas tasas de supervivencia muy favorables.

El balance global de este programa, después de 35 años de aplicación, es realmente impresionante: 700.000 ejemplares silvestres cazados, 5 millones de huevos recolectados, 2.7 millones de ejemplares criados en granjas y posteriormente comercializados...

En cuanto al recurso económico representado por el caimán, de él dependen los 2000 cazadores profesionales y el personal de las 55 granjas que existen en la actualidad. Los cálculos más conservadores estiman que la cifra de negocio ha alcanzado un mínimo de 495 millones de dólares en el periodo desde 1972, aunque otras evaluaciones más generosas estiman que el sector mueve unos 50 millones de dólares anuales. En cualquier caso, una cifra nada desdeñable, sobre todo si pensamos que la especie estuvo a punto de desaparecer.

Además, desde un punto de vista ecológico, el aprovechamiento inteligente del recurso ha permitido rentabilizar el ecosistema en el que vive la especie (una gran parte del cual es de propiedad privada) y ponerlo a salvo de otras utilizaciones mucho más agresivas, tan frecuentes en muchas zonas del mundo y que en muchos casos 216 han llevado a la desecación de estos humedales. 


\section{El efecto sinérgico de la recuperación de especies en peligro: los carneros de Pakistán}

En 1985, subsistían en el área de Torghar, en Pakistán, dos pequeñas poblaciones de carneros silvestres de alta montaña, el markhor (Capra falconeri), con menos de 100 ejemplares y el urial (Ovis vignei), con unos 200. Ambas poblaciones estaban al borde de la desaparición debido a una fuerte presión de caza de subsistencia por parte de cazadores locales y a la competencia excesiva con el ganado doméstico.

El programa de conservación se inició en 1986 mediante un acuerdo con los cazadores extranjeros, que accedieron a pagar casi 50.000 US\$ por 1 markhor y 4 uriales. Este dinero fue reinvertido en el desarrollo del programa y en la dotación de los medios humanos y materiales de los que carecían por completo los gestores locales.

Unos veinte años después, la población de markhor ha alcanzado la cifra de más de 2500, mientras que la de urial ha llegado a un mínimo de 3100. La utilización racional mediante la caza deportiva ha permitido que se hayan recreado las mayores poblaciones mundiales de estas especies, a pesar de la existencia de conflictos puntales con el ganado doméstico.

La caza ha generado, por sí sola, unos ingresos de 1,7 millones de US\$, de los cuales unos 300 mil han ido a parar a las arcas provinciales y el resto directamente a las comunidades locales.

Atraídos por este programa y su proyección internacional, organismos públicos (GEF, UNDP) y privados (Society for Torghar Environmental Protection, IUCN) han invertido también importantes cantidades de dinero (1.2 millones de \$) para formación de personal especializado (veterinarios, guías, etc.) así como en programas de mejora de las condiciones de vida de las poblaciones locales de esta región.

Aunque las cifras pueden parecernos modestas, estos presupuestos, significativos si se aplican en una de las áreas con mayor nivel de pobreza del planeta, han permitido no sólo la conservación de un recurso a punto de desaparecer sino que se han convertido en una posibilidad real y tangible de mejora de bienestar para las comunidades locales de la región.

La conservación de estas poblaciones de especies sumamente amenazadas, a través de la caza deportiva, ha supuesto un extraordinario ejemplo de la tan cacareada "utilización sostenible" de un recurso.

\section{CASO HIPOTÉTICO: ¿Y SI SE HUBIERA EXTINGUIDO UNA ESPECIE BANAL?}

\section{El tunicado Ecteinascidia turbinata}

Permítanme finalmente plantearles un supuesto que demuestra lo poco que sabemos de muchos seres vivos y, aún más, de sus potencialidades como fuente de 
materias primas para nuestra salud y nuestras economías. Y también de lo difícil que resulta calcular el beneficio que se podría perder con la desaparición de cualquier especie, poseedora de potenciales desconocidos.

La ascidia Ecteinascidia turbinata es un animal del Filum Tunicados (a veces llamados Urocordados, pues tienen cuerda dorsal, lo que los acerca a los vertebrados), un pequeño animal de unos pocos centímetros de longitud, que vive en colonias y se protege por una envoltura transparente similar a una túnica. Vive en mares templados o cálidos, como el Mediterráneo o el Caribe y no presenta especiales problemas de conservación, por lo que no hay nada que temer con respecto a su supervivencia.

Es decir, un "bicho" insignificante, uno de esos cientos de miles de organismos que viven en el mar y que pasan desapercibidos para casi todo el mundo, salvo para unos cuantos estudiosos.

Pero he aquí que nuestra insignificante ascidia tiene una peculiaridad extraordinaria: sintetiza un grupo de moléculas complejas denominadas ecteinascidinas, consideradas hoy en día como unas de las sustancias más prometedoras contra diversos tipos de tumores.

De una de estas moléculas, una empresa española de investigación biomédica, especializada en la búsqueda de nuevos compuestos a partir de organismos marinos, ha desarrollado, después de años de investigaciones, un nuevo medicamento antitumoral, un alcaloide sintético para el tratamiento de sarcomas de tejido blando.

Según la información que ofrece la empresa en su web, las ventas de este medicamento en Europa alcanzaron los 13,5 millones de euros en el año 2007 y se tienen unas expectativas de venta de hasta los 100 millones de euros en 2011, momento en el que se podría estar administrando a 4.000 pacientes. Esta cifra podría dispararse hasta los 1.200 millones de euros anuales en ventas a partir de 2012, si la Agencia Europea del Medicamento y la FDA americana autorizan su venta para el tratamiento del cáncer de ovario.

Aunque estas cifras hablan por sí mismas, el cálculo de las repercusiones económicas del desarrollo de este medicamento es mucho más complejo. ¿Cómo valorar el ahorro del gasto hospitalario que supondría la curación de parte de estos pacientes? ¿O en primas de seguro? ¿O en productividad en los años de vida ganados a la enfermedad? Por no hablar, por supuesto, de las repercusiones más importantes, no cuantificables económicamente pero si socialmente, relativas al sufrimiento humano de pacientes y sus familias.

Con estos ejemplos se ilustra le enorme valor que la protección de la biodiversidad tiene para la Humanidad, se ve la gran complejidad que encierra el análisis de

218 su pérdida y se muestra la imposibilidad de encontrar resultados concretos y preci- 
sos para todos los casos, al menos ahora y hasta que se disponga de una información más fiable.

Es evidente el largo camino que queda por recorrer en este campo y que aún serán necesarios muchos estudios y modelos que permitan entender mejor esta cuestión. Tal vez en ese momento, cuando podamos cuantificar el coste de la pérdida de biodiversidad de una manera mucho más eficiente y poner cifras al fenómeno de su pérdida, la conservación de la biodiversidad adquiera un mayor protagonismo para la sociedad y los gobiernos, y se dote a esa política de los recursos humanos y materiales de los que carece en la mayoría de los países, incluso los más avanzados. 
